\title{
Fast Fault Diagnosis Expert System for Rocket Launch
}

\author{
Qingzhen Zhang ${ }^{1}$ Wei Wang ${ }^{1}$ Lihui Song ${ }^{2}$ Zhang Ren $^{1}$ \\ ${ }^{1}$ School of Automation Science and Electronic Engineering, Bei Hang University, Beijing 100083, P. R. China \\ ${ }^{2}$ China Academy of Launch Vehicle Technology, Beijing 100076, P. R. China
}

\begin{abstract}
Nowadays, during rocket launch, the decision-making partly depend on the results of the rocket status inspection. But we know the fast and precisely detect and diagnose of rocket faults is a conflict problem in many cases of bulk information processing. In this paper three methods are proposed. First, a distributed system structure is built in order to save time of information transmission and processing. Then a hierarchical-reasoning strategy is introduced. The last method is on reasoning which using reverse and deep reasoning strategy. The proposed strategies have been applied in a prototype assistant decision-making system for rocket launch system.
\end{abstract}

Keywords: Rocket, Fault diagnosis expert system, Distributed structure, Knowledge base

\section{Introduction}

The safety of rocket launch, especially rockets with cosmonauts in, is very important for the cosmonauts, the economy and even the politics significance of the society. Because of the complexity of the whole rocket system, the accident of the rocket launch happened sometimes [1]-[3]. According to the statistical data, before the end of the year 1995, there are 249 times rocket flights in the whole world. But there are 166 failures, $38 \%$ of the failures happened in the launch phase. Rocket launch faults occupy the maximal proportion.

In order to make use of the field expert knowledge and the fault mode analyses results, detecting and evaluating the states of the rocket before the rocket launch is applied and approved an effective method to decrease the accidents [1]-[2]. This method has been used primarily in the Chinese rocket $\mathrm{C}-2 \mathrm{~F}$.

The decision-making of rocket launch depend on the information that the whole system status are in gear. This need precisely fault position and find the exact position quickly enough to meet the real time characteristic of the whole launch system. But the two requirements are conflictive in practice. To a fixed diagnosis skeleton system, when using more information, such as creasing the test points of the rocket will enhance the accuracy of the diagnosis, but it is likely to consume more system time. In the other side, if reducing the information, the high speed is pursued but the accuracy of the reasoning is lost.

In this paper, a distributed structure is proposed for fault diagnosis system in order to dwindle the time consume of information transmission and processing. Then a hierarchical-reasoning strategy is introduced. Last, proposed a reasoning strategy which using the reverse and deep reasoning strategy to find the exact fault position. Then the proposed strategies were applied in a prototype assistant decision-making system for rocket launch system.

\section{A distributed structure of the fault diagnosis system}

There are so many test points and complicated fault modes in the rocket system that it is difficult to use the centralized structure in the fault diagnosis system. Utilizing the structure of the distributed system, every subsystem detects the key equipments and diagnoses the key faults of its own part. It is much more fast and effective than a whole centralized system as in many other engineering environments.

Taking the carry-man rocket (CMR) of China for an example, figure 1 is the framework of the structure of the distributed fault diagnosis system.

In the figure 1, the inspection signals are in the data server. By the high speed and real time network, they are transported to the subsystems. The diagnosis subsystems complete the diagnosis task of their own. The whole diagnosis system works on the distribution and harmony of the diagnosis subsystem's task and completes system-level fault diagnosis. In further, it synthesizes the key info of all faults to give advice on the decision-making of rocket launch.

The structure has many merits. Through distributing and harmonizing the whole task for the subsystems, it reduces the scale of the knowledge base. Every subsystem has its own knowledge base. When there are complex system faults, it needs multi- 
subsystem to work together and the whole fault diagnosis system gives a diagnosis decision according the whole knowledge base which including complex expert experience about rocket faults. This structure increases not only the real-time characteristic, but also upgrades the accuracy of the fault diagnosis system of the rocket.

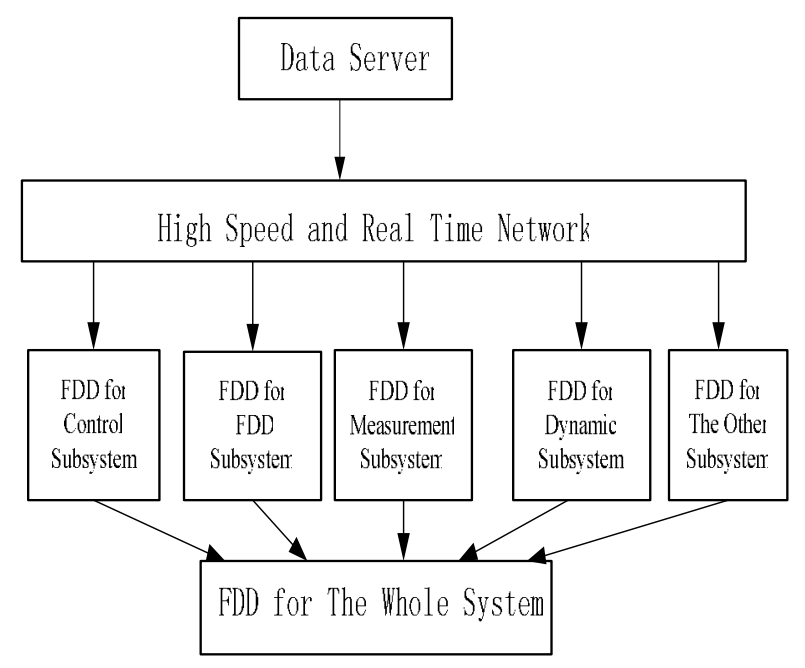

Figure 1: Structure of distributed diagnosis system for CMR.

\section{The fault hierarchical-reasoning strategy}

Because of the complexity of the rocket system and rich info involved in the inspection signals, the faults can be classified by significance. It can improve the efficiency and accuracy of the reasoning. Classifying the faults for 4 grades by significance, the reasoning matches the rules from the first grade to the fourth grade.

(1) The first grade named serious fault. They can lead directly failure of the rocket launch. The symptom information is from the measurement of the key part of the rocket. Since the amount of faults in this grade is very small, the reasoning is simple and the speed of the reasoning is also very fast. A few rules are used in this grade. Once one rule is matched, the conclusion 'The rocket is not allowed to launch' is presented immediately.

(2) The second grade is named key fault. When this grade fault happens, the rocket can also be launched sometimes after being carefully evaluated according to knowledge base.

(3)The third and the fourth grade fault is the low level fault. These grade faults need much more knowledge on basic devices or reasoning time. Further more, because of the inconspicuous influences to system; the reasoning and diagnosis of these faults will be proposed as a useful suggest.

\section{Reverse and deep reasoning strategies facing to the faults}

Because of the distributed structure and hierarchicalreasoning strategy of the fault diagnosis system, the system utilizes the reverse and deep reasoning strategy. Every subsystem has its own rule table; the whole fault diagnosis system also has its own system level rule table. The rules are matched from high grade to low grade and the same grade rules are traversed according to storage sequence.

The reasoning process is presented by the rule table in figure 2 .

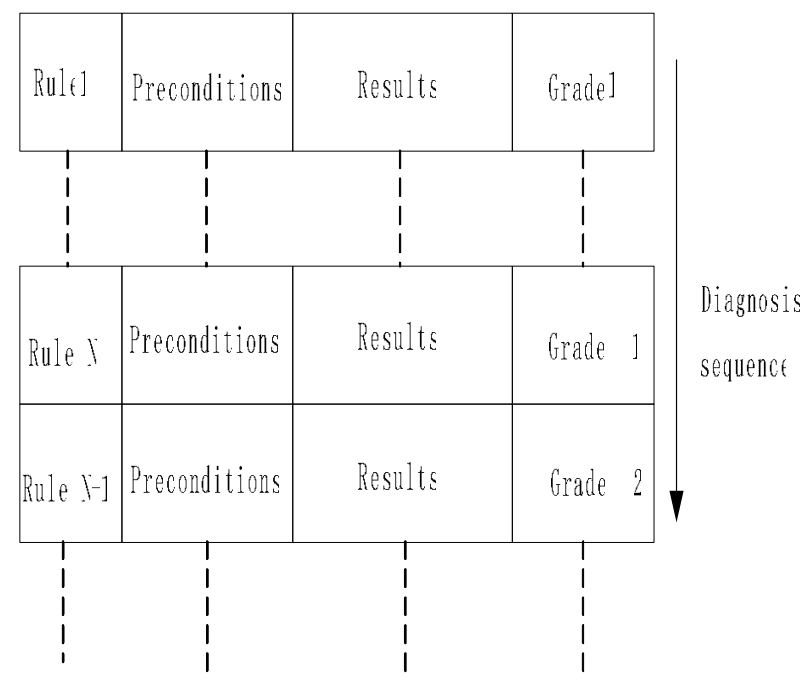

Figure 2: Reasoning process.

For the convenience of constructing the rule table, the order of the rules of the same grade fault is random. Since the preconditions of a rule may be the conclusions of the other rules, when only simply traversing the rule table in the same grade, some of the rules will not be matched. This will result in some faults be neglected.

This paper gives a deep reasoning algorithm to solve the problem. Once any rule is matched, the reasoning algorithm will be activated. This algorithm will run in every subsystem individually.

Figure 3 shows the flow chart of the deep reasoning algorithm. The key idea of this algorithm is the reasoning algorithm will be over if and only if no rule is matched successfully. 


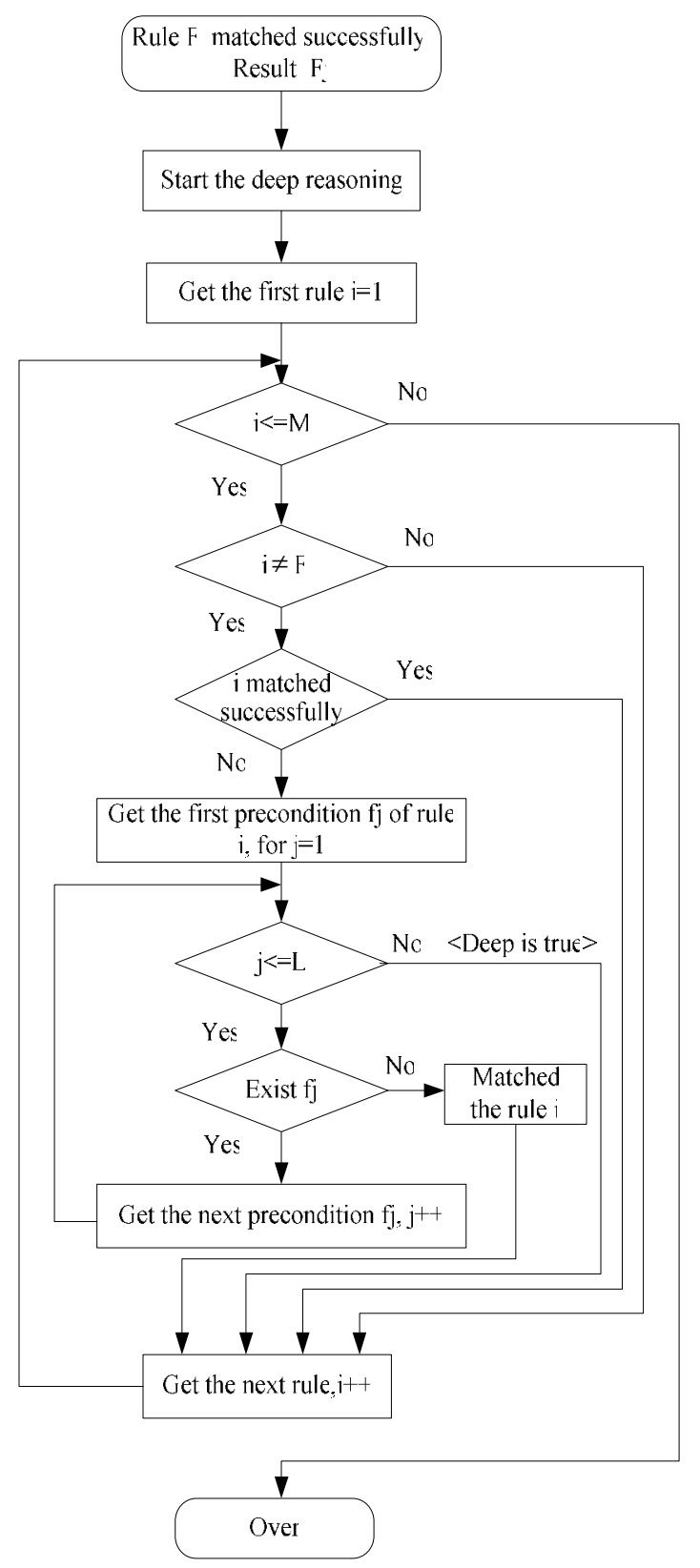

Figure 3: The flow chart of the deep reasoning algorithm.

In the algorithm, the rules are denoted as integers, so one given integer means one rule. These integers are stored in order. Also the conclusions and preconditions are also stored as integers. $\mathrm{F}$ is a matched integer; $\mathrm{M}$ is the maximal integer in the rule table. ' $i \leq M$ 'means the algorithm should transverse the whole rule table. $\mathrm{Fj}$ is a conclusion; $\mathrm{L}$ is the maximal number in the precondition table. ' $j \leq L$ 'means the algorithm should transverse the whole precondition table. The key idea of this algorithm is the reasoning algorithm will be over if and only if no rule is matched successfully.

By the method introduced in this paper, based on $\mathrm{C}++$ Builder development environment, a prototype FDD system is constructed for the escape subsystem of a Chinese carry-man rocket .It contains 58 items info of test points and 34 rules, and it can diagnose 27 faults. A test point fault diagnosis report of the escape subsystem is given in appendix. The report proves the accuracy and validity of these methods.

\section{Conclusions}

This paper researches on fast fault detection and diagnosis for rocket launch. It provides three methods to complete the fast decision-making: the distributed structure of the synthetically diagnosis system, the hierarchical-reasoning strategy and reverse and deep reasoning strategies. The diagnosis report of a prototype fault diagnosis system for the escape subsystem of a Chinese carry-man rocket proves the validity and the accuracy of the proposed method.

\section{Acknowledgement}

This work is partially supported by National Nature Science Foundation of China (Grant No. 60234010). The authors would like to acknowledge post doctor Chengrui Liu of Bei Hang University for his helpful suggestions during the early part of this work. The authors would like to acknowledge doctor Qingdong $\mathrm{Li}$ of the Northwestern Polytechnical University for providing a specialized searching module for the reference engine of expert system used in this work. The authors would like to acknowledge Professor K.M Zhou of Louisiana State University for providing helpful insight throughout the course of this work.

\section{References}

[1] Q.X. Zhou, H.L. Guo, The research prospect on fault characteristics and fult diagnosis techniques for the carry-man launch vehicle. Aerospace China, 9:30-32,1999.

[2] X. Yang, etc., Survey of fault diagnosis and faulttoleran control technology for spacecraft. Journal of Astronautics, 24:221-226,2003.

[3] B. Wang, etc., Inference Engine Designed for Real-time Monitor and Launching Decisionmaking Expert System. Journal of System Simulation, 16:820-822,2003.

[4] C.Q. Yang, H. Yang, AI and Expert System, China Water Power Press, Bei Jing, 2002.

[5] P. M. Frank, Fault diagnosis in dynamic systems using analytical and knowledge-based 
redundancy-a survey and some new results. Automatica Control,26:459-474, 1990.

[6] J. J. Gertler, Survey of model-based failure detection and isolation in complex plants. IEEE Contr. Syst.Mag, 8: 3-11, 1988.

[7] R. Isermann, Process fault detection based on modeling and estimation methods: a survey. Automatica Control, 20: 387-404, 1984.

[8] K.M Zhou, Z. Ren, A new controller architecture for high performance, robust and fault-tolerant control. IEEE Transactions on Automatic Control, 46(10) 1613-1618, 2001.

[9] D.U. Campos-Delgado, K.M. Zhou, Reconfigurable fault-tolerant control using GIMC structure. IEEE Transactions on Automatic Control, 48: 832-838, 2003.

[10] J.R. Noriega, H. W, A direct adaptive neuralnetwork control for unknown nonlinear systems and its application. IEEE Transactions on Neural Networks, 9: 27-34, 1998.

[11] R.J. Veillette, J.V.Medanic, W.R.Perkins, Robust stabilization and disturbance rejection for systems with structureduncertainty. Proc.28th IEEE Conf. Decision Contr., pp. 936-941, 1989.

[12] R.J.Veillette, J.V.Medanic, W.R.Perkins, Design of reliable control system. IEEE Trans on Automatic Control, pp.290-304, 1992.

[13] X. Yan, H.X. Wu, X.L. Wang and Z.B. Li, Survey of fault diagnosis and fault-tolerance control technology for spacecraft. Journal of Astronautics, 24: 221-226, 2003.

[14] Y. Zhang, Z.W. Zhang, X.C. Dong, Design and Implementation of Real-Time Diagnostic Expert System in Nuclear Power Plant. Atomic Energy Science and Technology, 40(4): 420-423, 2006.

[15] J. C. Giarratano, Gary D. Riley, Expert System: principles and programming. PWP Publishing Company, Bei Jing, 1998.

[16] R.J. Patton, I. Chen, Fuzzy observers for nonlinear dynamic system's fault diagnosis. Proceedings of the 37th IEEE Conference on Decision \& Control, pp. 84-89, 1998.

[17] H. Wang, Fault detection and diagnosis for unknown nonlinear systems: A generalized framework via neural networks. In Proceedings of the IEEE International Conference on Intelligent Processing Systems, pp: 1506-1510, 1997.

[18] J D. Boskvic, Evaluation of the Properties of a Multiple-model Reconfigurable Flight Controller on a 6 DOF Simulation. AIAA, 2000-4039, pp. 285-295, 2000.

[19] P. Kabore, H. Wang, Design of fault diagnosis filters and fault tolerant control for a class of nonlinear system. IEEE transactions on automatic control, 46(11): 1805-1810, 2001.

[20] D.H. Zhou, Y.G. Xi, Z.J. Zhang, A suboptimal multiple fading extended Kalman filter. Chinese Journal of Automation, 4:145-152,1992.

\section{Appendix}

Below is part of diagnosis report of the escape subsystem of a Chinese carry-man rocket, and the report can demonstrated the system's reasoning process.

The input info from the test points:

(1) The voltage of the test points from light terminal to the assistant control computer cable is $5 \mathrm{~V}$;

(2) Run state indicator light does not blink timely;

(3) There is no electric current in the test points;

(4) Not receiving the light signals.

Test results:

※---diagnosis results--- ※

Fault one:

result number: №2

preconditions of the result:

(1) The voltage of the test points from light terminal to the assistant control computer cable is $5 \mathrm{~V}$;

(2)Run state indicator light does not blink timely.

\section{result}

The collection board of the main measurement computer on the ground is faulty.

The influence of the result to the system is :

The ground centre can't watch the run status of the software in the launch vehicle.

The grade of the fault: grade two.

$* * * * * * * * * * * * * * * * * * * * * * * * * * * * * * * * *$

Fault two:

result number: №4

preconditions of the result:

(1)Run state indicator light does not blink timely;

(2) Not receiving the light signals.

result

The light fiber between the two light terminal occurs fault.

The influence of the result to the system is :

The ground centre can't watch the run status of the software in the launch vehicle.

The grade of the fault: grade two.

***************************************

Fault three:

result number: №6 
preconditions of the result:

(1)Run state indicator light does not blink timely;

(2) There is no electric current in the test points.

$\diamond$ result

The cable between the light terminal and launch

vehicle occurs fault.

The influence of the result to system is :

The ground centre can't watch the run status of the software in the launch vehicle.

The grade of the fault : grade two. 